\title{
Research Paper \\ Psychometric Properties of the Iranian Version of the Coronavirus Anxiety Scale
}

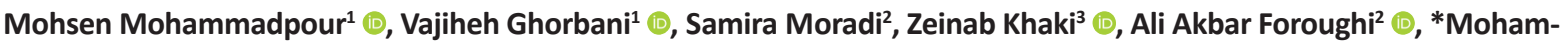 \\ mad Reza Rezaei ${ }^{4}$ [C
}

1. Department of clinical psychology, School of Behavioral Sciences and Health Mental (Psychiatry of Institute Tehran), Iran University of Medical sciences, Iran, Tehran 2. Department of Psychology Clinical, School of Medicine, Kermanshah University of Medical Sciences, Kermanshah, Iran.

3. Department of Psychology, School of Medicine (Kermanshah Science and Research International Campus), Islamic Azad University, Kermanshah Branch, Kermanshah, Iran. 4. Department of Emergency Medicine, School of Medicine, Kermanshah University of Medical Sciences, Kermanshah, Iran.

\begin{tabular}{|c|c|}
\hline $\begin{array}{l}\text { Use your device to scan } \\
\text { and read the article online }\end{array}$ & Cttation Mohammadpour M, Ghorbani V, Moradi S, Khaki Z, Foroughi AA, Rezaei MR. Psychometric Properties of The \\
\hline 口ising & $\begin{array}{l}\text { Iranian Version of The Coronavirus Anxiety Scale. Iranian Journal of Psychiatry and Clinical Psychology. 2020; 26(3):374-387. } \\
\text { http://dx.doi.org/10.32598/ijpcp.26.3482.1 }\end{array}$ \\
\hline 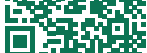 & doil'http://dx.doi.org/10.32598/ijpcp.26.3482.1 \\
\hline
\end{tabular}

Received: 19 June 2020 Accepted: 27 Oct 2020 Available Online: 01 Dec 2020

Key words: Validity and reliability Confirmatory factor analysis, Coronavirus anxiety scale

\begin{abstract}
Objectives In the context of the epidemic of coronavirus and its psychological effect, it is necessary to develop appropriate tools for measuring the aspects of its psychological pathology. Therefore, this study aims to determine coronavirus anxietyss psychometric properties using a 5-point Likert scale (Sherman A. Lee) in the Iranian statistical population.

Methods In this study, 399 men and women from the adult population of Kermanshah were studied by the available sampling method. The Fear of COVID-19 Scale (FCV-19S), Difficulties in Emotion Regulation Scale(DERS), as well as the second version of the Acceptance and Action Questionnaire (AAQ-II) were used for convergent validity and divergent validity, respectively. Internal consistency method using Cronbach's alpha was used to analyze data, and confirmatory factor analysis using Lisrel-8.8 software was used for construct validity.

Results Cronbach's alpha was found to be 91.5 for the scales overall score. CAS found a positive and significant correlation between the coronavirus anxiety scale and the sub-components of difficulty in emotion regulation (except for problems engaging in goal-directed behaviors). Still, he also found that there was a negative correlation between acceptance and action. Furthermore, the results of the confirmatory factor analysis indicated that the single-factor structure is well-suited

Conclusion Covid-19 related anxiety can be disproportionate and unnecessary and cause many psychological problems. The Iranian version of the coronavirus anxiety Scale reflected desirable validity and reliability and could be used as a short and valid screening tool for measuring coronavirus anxiety measurement.
\end{abstract}

\section{Extended Abstract}

\section{Introduction}

oronavirus disease first broke out in $\mathrm{Wu}$ han, China, in December 2019 [1]. Today, most countries globally, including Iran, are thinking of controlling the disease's spread through social distance and observing health protocols. Still, little attention has been paid to its psychological aspects [6-8]. Anxiety is considered a common symptom among patients with chronic respiratory disorders and could significantly reduce patients' quality of life [13]. Regarding coronavirus, anxi-

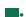

* Corresponding Author:

Mohammad Reza Rezaei, PhD.

Address: Department of Emergency Medicine, School of Medicine, Kermanshah University of Medical Sciences, Kermanshah, Iran.

Tel: +98 (935) 7914475

E-mail: mohammadreza.rezaei@kums.ac.ir 
ety is common among people because it is unknown and undecided $[14,15]$.

Similarly, different tools have been developed to measure chronic respiratory disorders like SARS and MERS in the past. In most cases, anxiety measurement also included physical symptoms due to chronic respiratory diseases and medications' side effects $[13,20,21]$. It is necessary to create tools to identify anxiety related to this disease and the strategies to prevent it from spreading in the current situation. With the onset of coronavirus, Lee [26] developed a mental health screening tool related to coronavirus. However, considering its limitations, it is necessary to explore its psychological characteristics across the world.

Therefore, a valid and short tool is needed to measure anxiety related to coronavirus due to the increasing anxiety and concern among people because of the impact of this virus on their various functions such as occupation and education. Thus, this study aims to determine the coronavirus anxiety scale's psychometric characteristics in the Iranian statistical population.

\section{Method}

The participants in this study consisted of 399 adults from Kermanshah city who participated online in this study from May 4 to May 7. The authors also obtained the informed consent of participants to take part in this study before filling out demographic information. CAS was conducted based on guidelines needed to standardize instruments in different cultures $[33,34]$.

\section{Instruments}

Coronavirus anxiety scale: this scale includes 5 questions that measure cognitive, behavioral, emotional and physiological dimensions related to coronavirus anxiety during the past two weeks. The questions are scored based on a 5-point Likert scale from 0 (Never) to 4 (Almost every day) [26].

The Fear of COVID-19 Scale (FCV-19S): This scale was developed by Kwasi Ahorsu et al. to examine the fear of coronavirus by people. A higher score indicates more fear of coronavirus. Internal consistency and retest reliability were found to be 0.82 and 0.72 , respectively [6].
Difficulties in Emotion Regulation Scale (DERS): DERS is a self-report tool consisting of 36 materials that measure the normal levels of difficulty in emotion regulation. The whole main scale's reliability coefficient and the retest reliability were reported to be 0.93 and 0.88 , respectively, and the construct and predictive validity were reported to be desirable [35]. This scale was normalized in Iran by Khanzadeh et al. [36].

Second version of the Acceptance and Action Questionnaire (AAQ-II): This questionnaire measures the construct associated with diversity, acceptance, experiential avoidance, and psychological flexibility. Higher scores suggest greater mental flexibility. The mean alpha coefficient was found to be 0.84 , and retest reliability, in 3 to 12 months, was found to be 0.81 and 0.79 , respectively [37]. This instrument in Iran has appropriate psychometric features [39].

\section{Statistical analysis}

Data were analyzed using SPSS software V. 25 and Lisrel software V. 8.8. Descriptive statistics were used to analyze data, and Cronbach's alpha was used to examine internal consistency. Confirmatory factor analysis was also used to determine to construct validity using Lisrel software V. 8.8.

\section{Results}

This study was conducted on 399 people with a mean age of $31.57 \pm 9.74$. Cronbach's alpha was used to analyze validity. The results of this study suggested that internal consistency using Cronbach's alpha equals 91.5.

The correlation between the coronavirus anxiety scale and the Fear of COVID-19 Scale, difficulty in emotion regulation questionnaire, and acceptance and action questionnaire were analyzed to determine validity. The correlation between CAS and FCV-19S and the correlation between CAS and the overall score of DERS were found to be 0.45 and 0.27 , respectively, which indicates favorable convergence validity of this scale. Moreover, the coronavirus anxiety scale negatively correlates with AAQ-II $(r=0.43)$, suggesting its desirable divergence validity.

Confirmatory factor analysis was used to analyze the validity of the construct. The results of this study indicated that

Table 1. Fit indexes for CAS

\begin{tabular}{ccccccc}
\hline Fit Indexes & $\boldsymbol{\chi}^{2} / \mathbf{d f}$ & $\mathbf{p}$ & GFI & NFI & CFI & RAMSE \\
\hline One factor & 1.61 & 0.18 & 0.99 & 1 & 1 & 0.04 \\
\hline & & & & & $\begin{array}{c}\text { Iranian Journa of } \\
\text { PSYCHIATRY AND CLINICAL PSYCHOLOGY }\end{array}$
\end{tabular}


the single-factor model has proper fitting $(\mathrm{P}=0.18, \mathrm{df}=2$, chisquare $\left.=4.83, \mathrm{RMSEA}=0.04, \chi^{2} / \mathrm{df}=1.61\right)$. Table 1 shows the results of this study.

\section{Discussion and Conclusion}

CAS showed proper internal consistency, and its Cronbach's alpha was found to be 91.5 . These results are consistent with those of Lee [28], who reported that Cronbach's alpha for coronavirus anxiety scale is 0.93 . The convergence validity results indicated that CAS has a positive and significant correlation with FCV-19S, indicating that those who are more afraid of coronavirus experience more anxiety. This finding supports the work of other studies $[6,14,28]$. Also, all of the sub-components of difficulty in emotion regulation(except for difficulties engaging in goal-directed behaviors) had a positive and significant relationship with CAS. The problems of emotion regulation have been identified in many Axis I and Axis II disorders [43, 44]. Emotional dysregulation has a significant relationship, especially with depression and anxiety disorders [47, 48]. Thus, it could be argued that those who can hardly regulate their emotions in response to coronavirus disease will experience more anxiety.

The results of divergence validity also indicated that experiential avoidance had a negative relationship with CAS. The growing body of literature has shown that those with low flexibility reported higher depression and anxiety levels $[51,54]$. These findings agree with other studies in this area, analyzing the psychological aspects of coronavirus [55-57]. The results related to constructing validity also revealed that the single-factor construct has a proper fitting, according to the initial studies [26]. This study had a few limitations, namely convenience sampling and brief analysis by WhatsApp.

Finally, CAS showed proper psychometric characteristics, and health care professionals could use this screening tool to detect coronavirus-related anxiety, which might last for months.

\section{Ethical Considerations}

\section{Compliance with ethical guidelines}

This research has been approved in Kermanshah University of Medical Sciences with the Code 3010137.

\section{Funding}

This article is taken from the Mentor Plan approved by Kermanshah University of Medical Sciences.

\section{Authors' contributions}

Conceptualization: Mohsen mohammadpour, Ali akbar foroughi; Methodology: Mohammad reza Rezaei, Samira Moradi; Investigation, sources, preparation, writing-review \& editin: Mohsen mohammadpour, Vajiheh Ghorbani; Validation, analysis, Project administration: All authors.

\section{Conflicts of interest}

This authors declared no conflict of interest

\section{Acknowledgements}

The authors would link to thank the Research and Technology Center of Kermanshah University of Medical Sciences and all health advocates. 


\title{
ويثَّى هاى روانسنجى نسخه ايرانى مقياس اضطراب كرونا
}

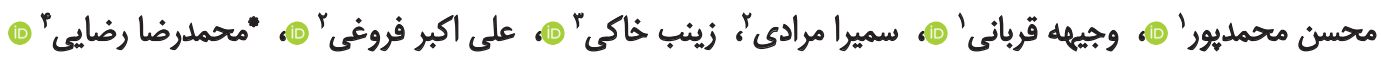

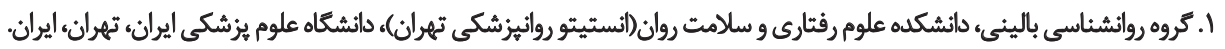

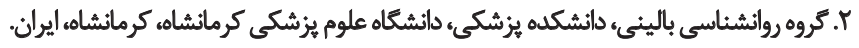

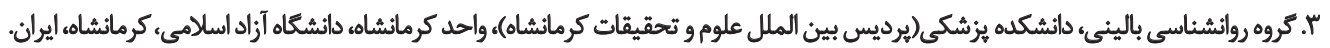

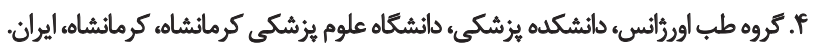

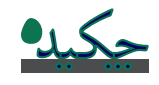

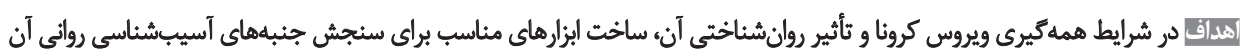

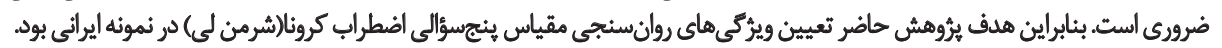

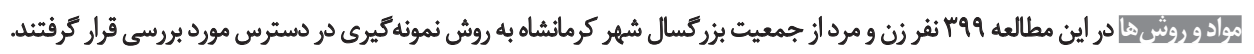

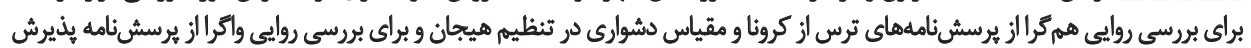

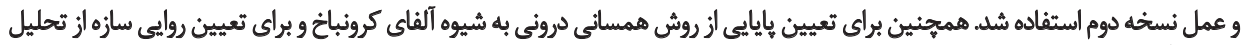

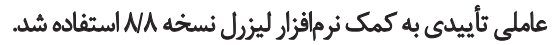

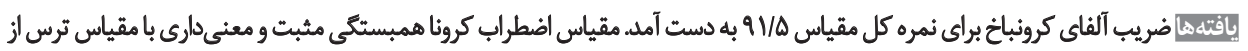

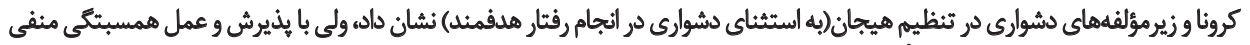

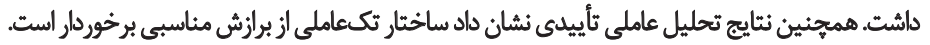

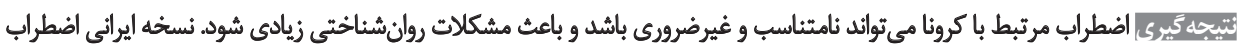

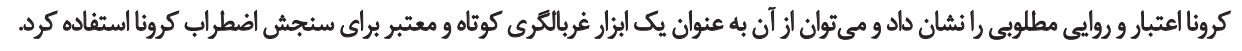

تاريخ دريافت: •"ماهرداد

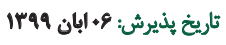

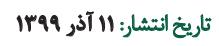

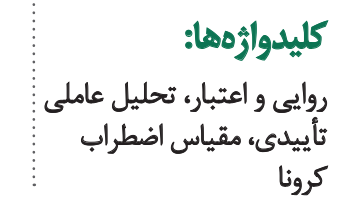

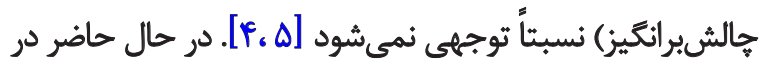
dove

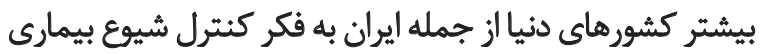

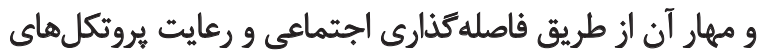

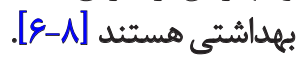

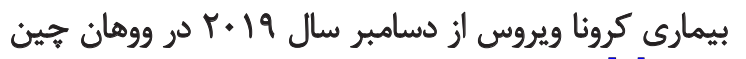

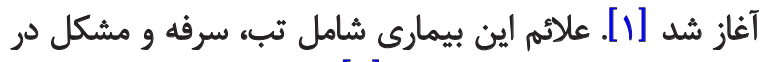

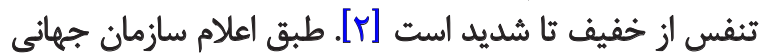
تحقيقات در مورد شيوع بيمارى هاي جهاني قبلي نشان دادهاند

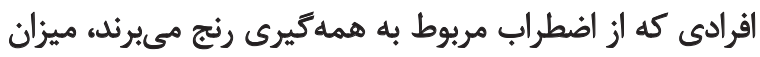

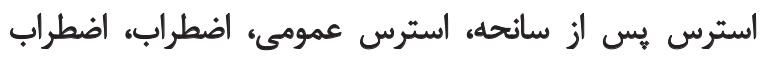

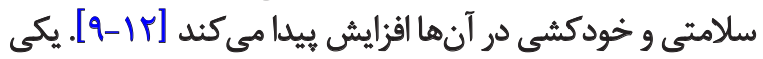

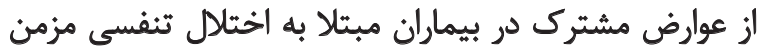

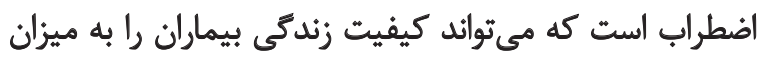

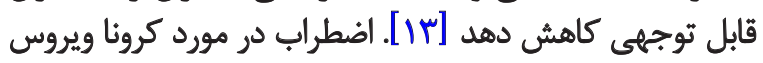

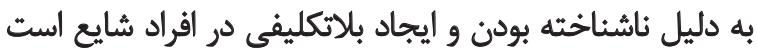

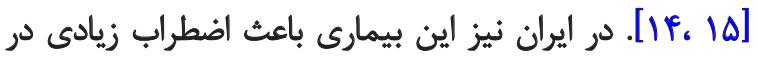

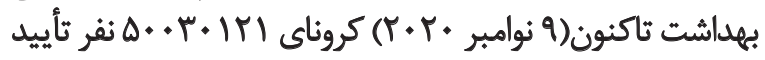

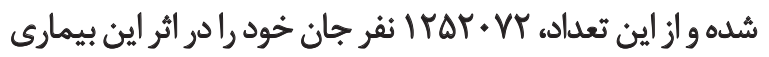

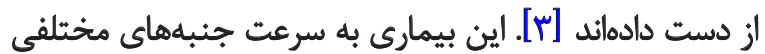

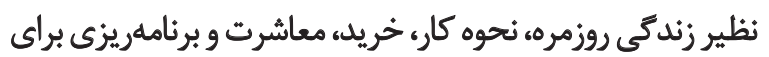

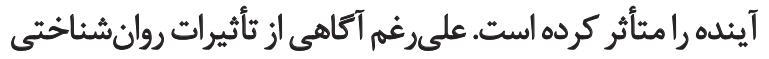

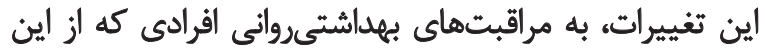

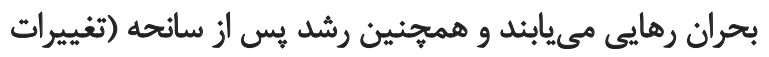

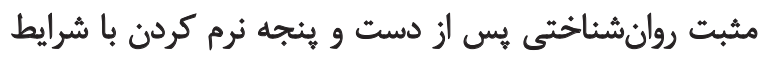




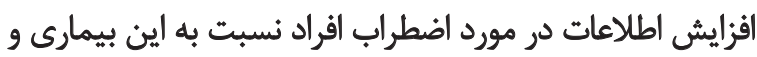

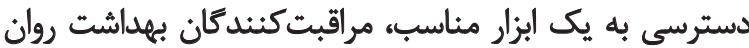

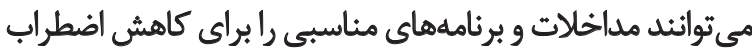

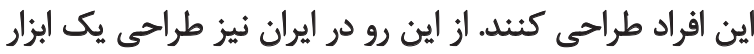

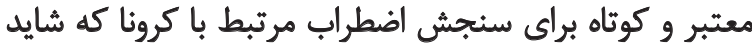

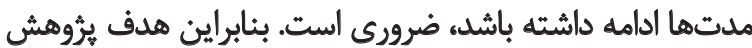

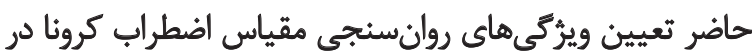

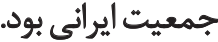

وروش

جامعه اين بُروهش، و 9 نفر از بزركسالان شهر كرمانشاه بودند

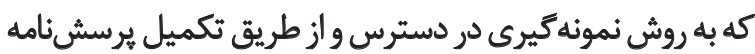

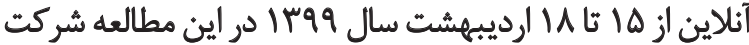

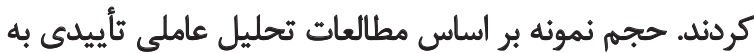

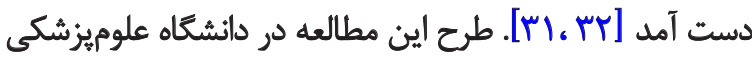

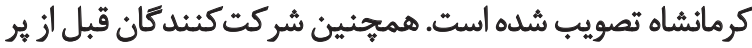

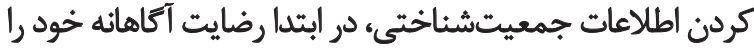

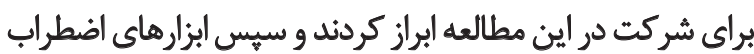

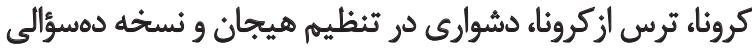
يرسشنامه بذيرش و عمل را تكميل كردند.

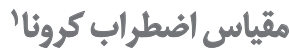

اين مقياس داراي بنج سؤال است كه ابعاد شناختى، رفتارى،

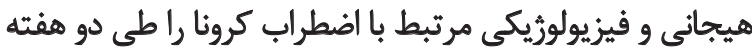

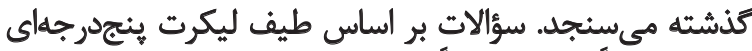

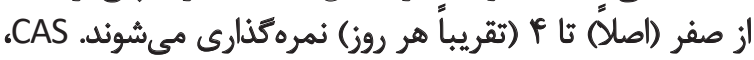

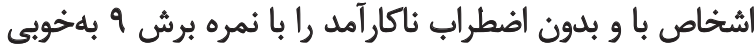

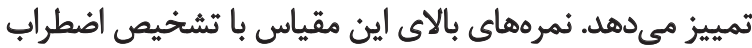

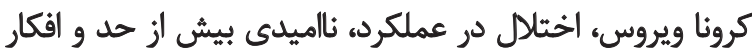

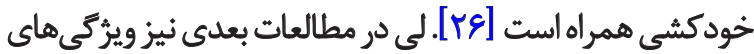

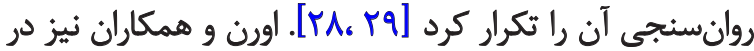

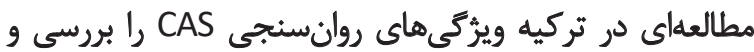

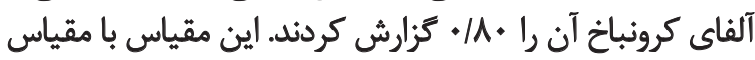

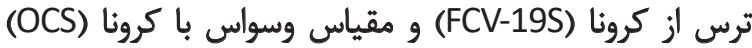

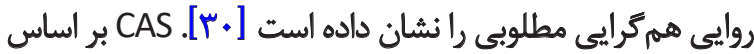

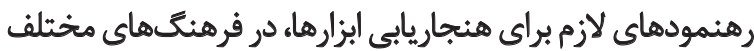

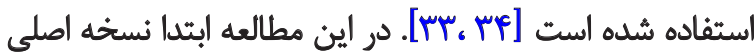

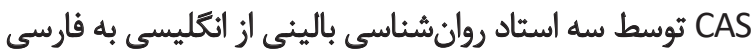

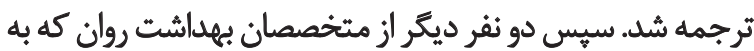

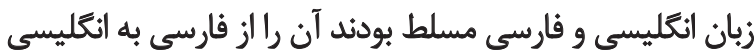

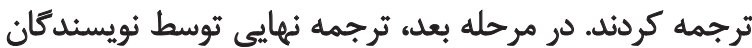

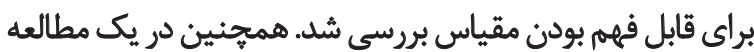

1. Coronavirus Anxiety Scale (CAS)

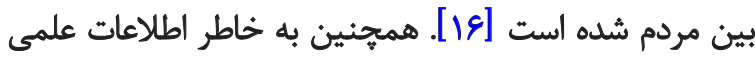

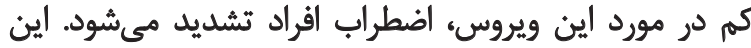

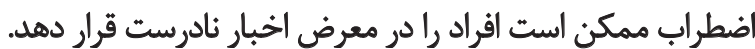

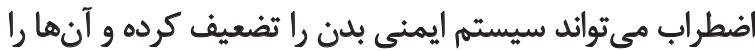

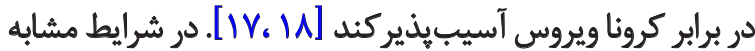
در كذشته ابزارهاى مختلفى براى سنج

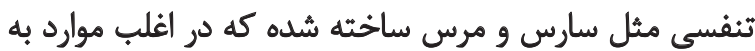

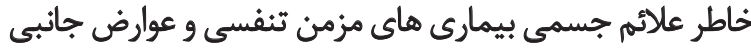

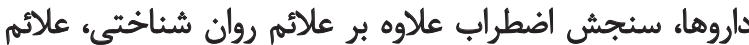

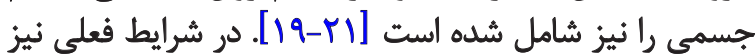

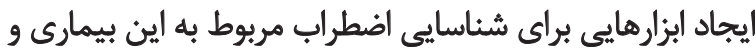

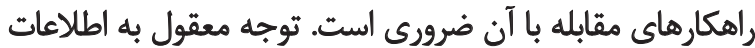

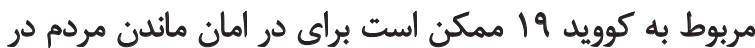

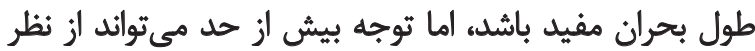

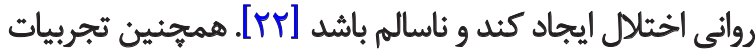

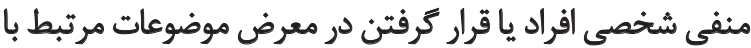

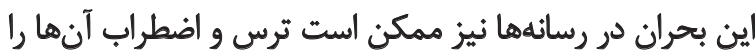

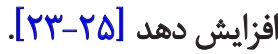

در مطالعه اخير بين VVD بزرتسال مقيم ايلات متحده

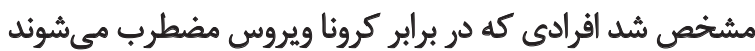

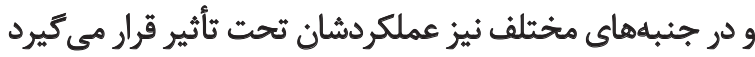

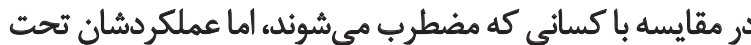

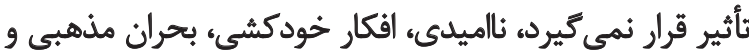

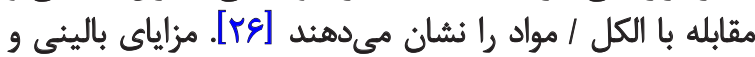

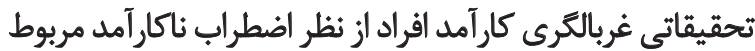

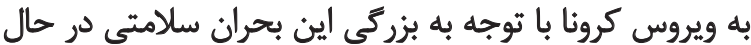

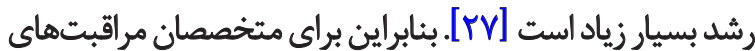

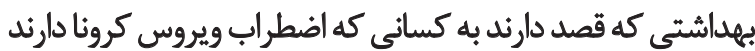

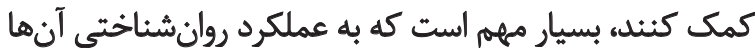

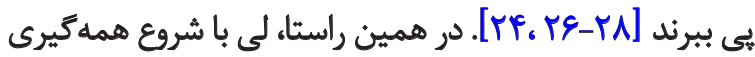

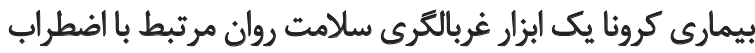

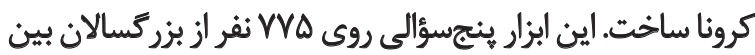

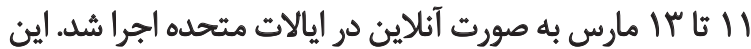

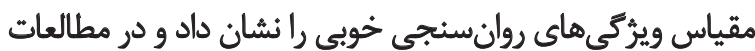

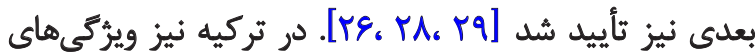

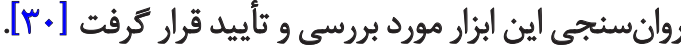

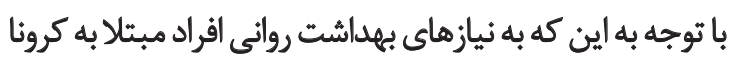

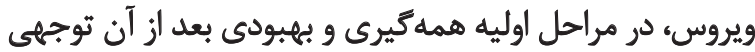

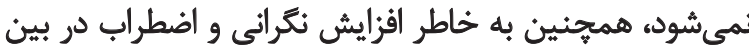

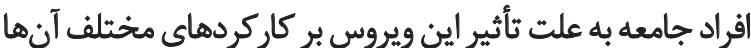

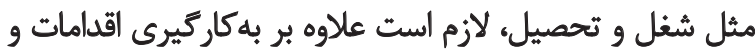

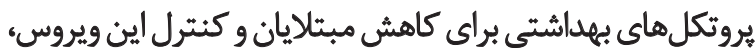

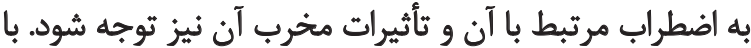


نسخه اول اين ثبرسشنامه توسط هيز و همكاران در سال

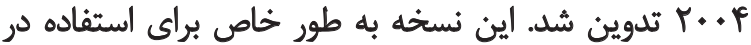

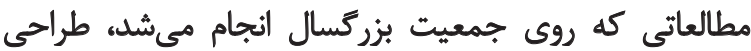

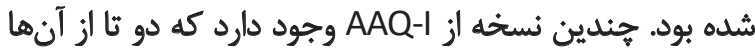

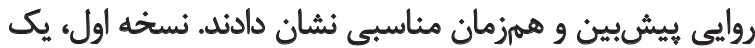

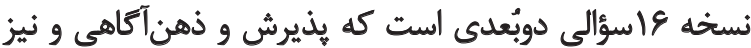

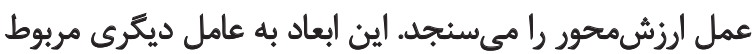

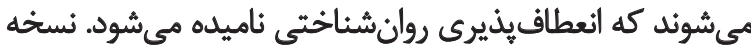

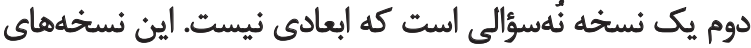

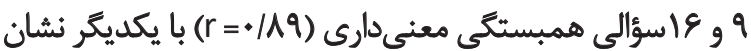

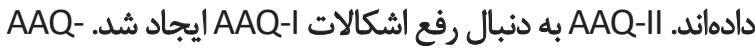

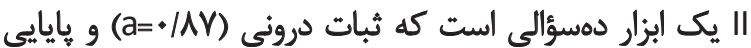

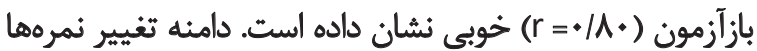

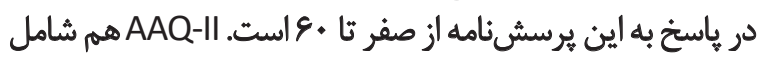

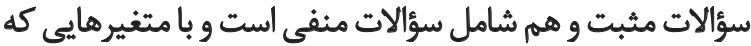

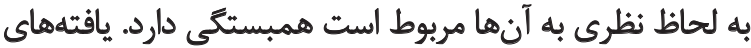

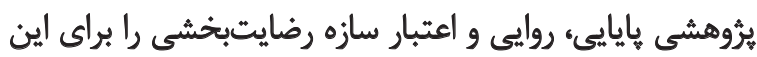

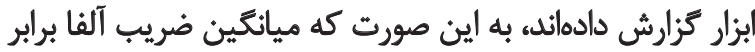

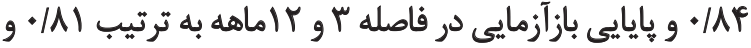

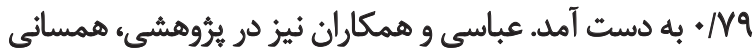

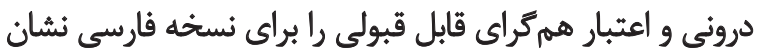

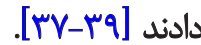

$$
\text { تونليل أماري }
$$

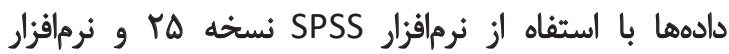

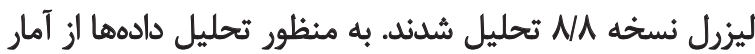

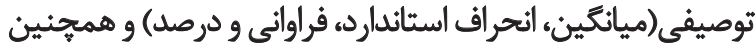

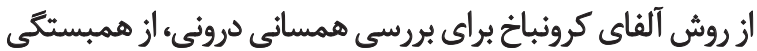

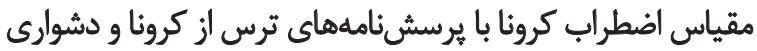

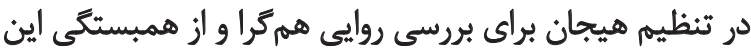

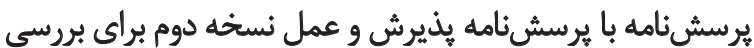

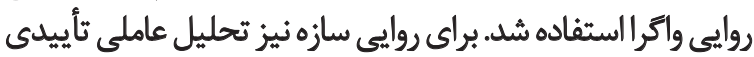

به كمك نرمافزار ليزرل نسخه N/N مورد استفاده قرار كرفت

يافتهها

اين مطالعه روى

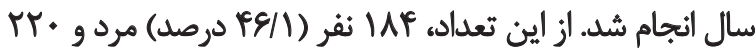

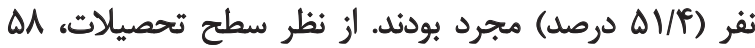

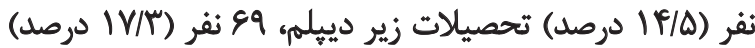

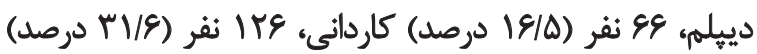

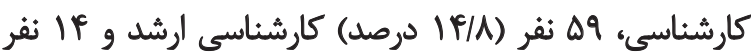

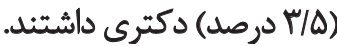

مقدماتى مقياس روى ها نفر اجرا شد و مشكلات سؤالها اصلاح شندئ.

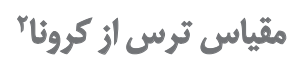

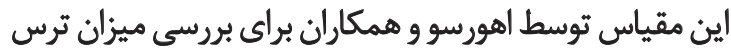

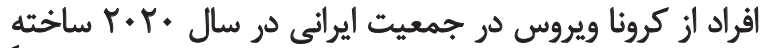

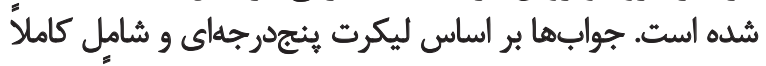

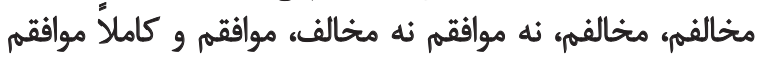

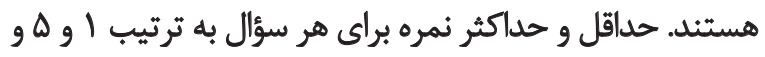

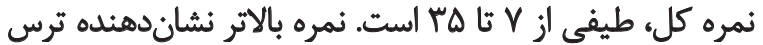

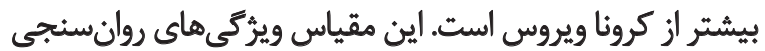

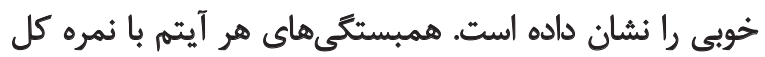

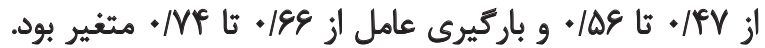

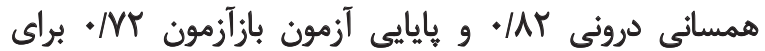

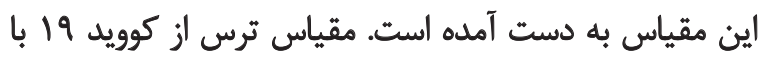

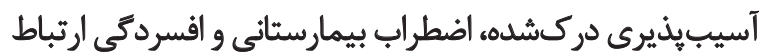
مثبت داشت [ع]. در مطالعه فعلى آلفاي كرونباخ اين مقياس آسياس

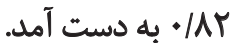

مقياس دشوارى در تنظيم هيجان

اين مقياس يك ابزار خودكزارشى عآمادماي است كه سطوح

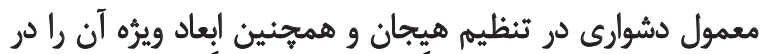

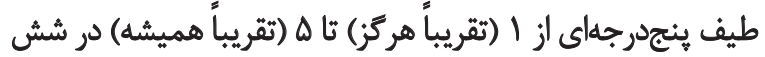

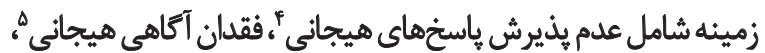

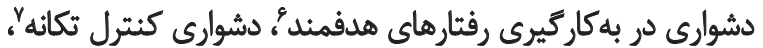

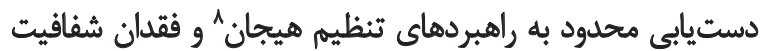

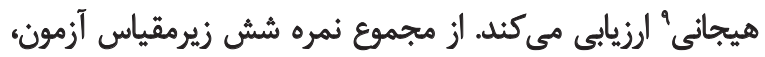

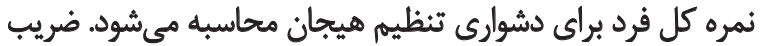

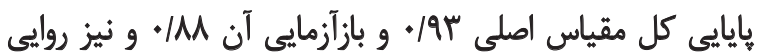

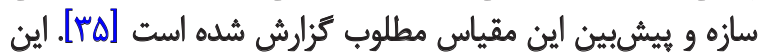

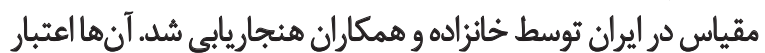

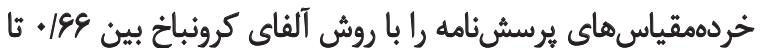

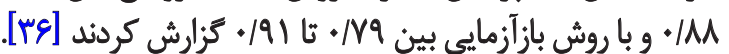
برسش نامه بذايرش و عمل نسخه دوم"1.

2. The Fear of COVID-19 Scale (FCV-19S)

3. Diffiiculties in Emotion Regulation Scale (DERS)

4. Non-acceptance of emotional responses

5. Lack of emotion awareness

6. Difficulties engaging in goal-directed behaviors

7. Impulse control difficulties

8. Limited access to emotion regulation strategies

9. Lack of emotional clarity

10. Acceptance and Action Questionnaire-II (AAQ-II) 
جدول ا. بررسى تحليل كويههاي مقياس اضطراب كرونا

\begin{tabular}{|c|c|c|c|c|}
\hline \multirow{2}{*}{ صورت حذف كويل } & \multirow{2}{*}{ ها بمرتى كلويهها } & \multirow{2}{*}{ صورت حذف موياس در } & \multirow{2}{*}{ ميورت حذف مقياس دويه } & كويهها \\
\hline & & & & طى دو هذته كذشته \\
\hline$\cdot / 9 \cdot r$ & $\cdot M T V$ & $11 / 9 \cdot 1$ & $r / M V$ & هنكام خواندن يا شنيدن خبرهاي مربوط به كرونا، احساس كيجى، \\
\hline - IMA & - Alr & $1 . /$ AFE & $r / l$. & در به خواب رفتن يا تلدوم خواب مشكل داشتم جون به كرونا \\
\hline .1199 & . NAV & $\mid r / T e$. & $r / r$. & 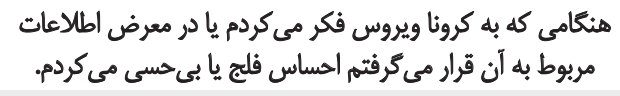 \\
\hline - IMA & $+/ A+V$ & $11 / 481$ & $r / r)$ & 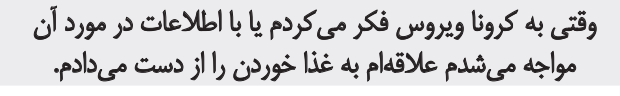 \\
\hline.$/ 19$. & $\cdot / \mathrm{YM}$ & 佂 & $r / Y V$ & 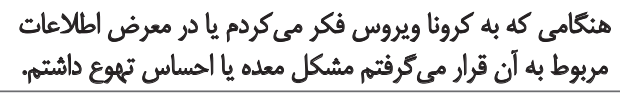 \\
\hline
\end{tabular}

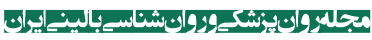

جدول Y. همبسثكي مقياس كرونا با ترس از كرونا، يرسشئامه تنظيم هيجائي و زيرمقياسهاي آن و برسشئامه بذيرش و عمل

\begin{tabular}{|c|c|}
\hline ضريب همبستكى & مثغيرها \\
\hline.$/ 40^{*}$ & مقياس ترس از كرونا \\
\hline.$/ T W *$ & مقياس دشوارى در تنظيم هيجان (نمره كل) \\
\hline - Mffe & زيرمقياس عدم هئيرش ياسخخهاى هيجائى \\
\hline.$/ Q$ & زيرمقياس دشوارى در ائجام رفتار هذفمند \\
\hline - /NeE" & زيرمقياس دشوارى در كتثرل تكانه \\
\hline$+/ \aleph^{*}$ & زيرمقياس فقدان كنترل هيجانى \\
\hline$+/ \mu \varphi^{*}$ & زيرمقياس دسترسى محلود به راهبردهاى تنظيم هيجانى \\
\hline$+/ 411 \%$ & زيرمقياس عدم وضوح هيجانى \\
\hline$-. / 4+4 \phi^{*}$ & مقياس يذيرش و عمل \\
\hline
\end{tabular}

$$
\text { تحاليل عاملي ثأيبدى شماره (ج). }
$$

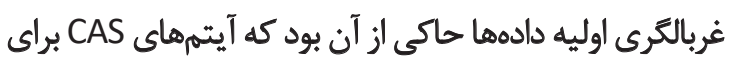

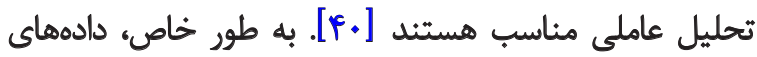

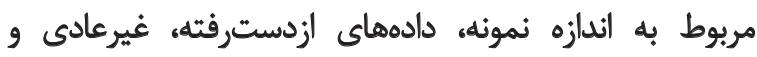

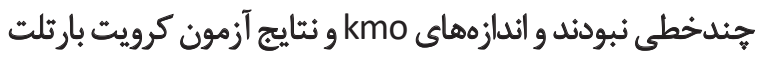

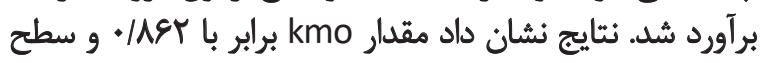

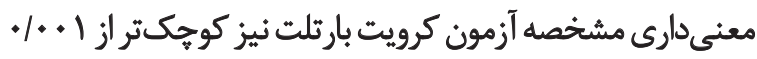

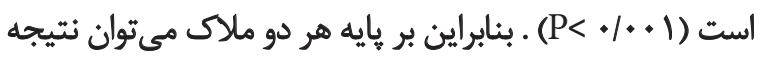

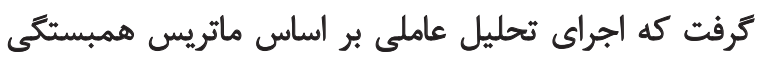
حاصل در كروه نمونه مورد مطالعه قابل توجيه است استي
ياياينى

همساني درونى به روش آلفاي كرونباخ برابر با 91/ه به دست

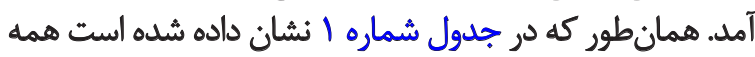
آيتمها در صورث حذف باعث كاهش ميزان آلفا مي شيوند كه نشان دهنده اين است كه آيتمهها مناسب هستئد

$$
\text { روائي همئراو واكرا }
$$

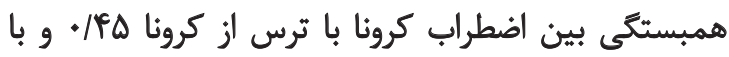

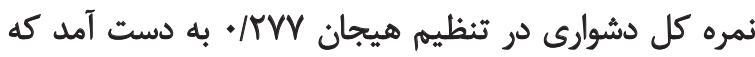

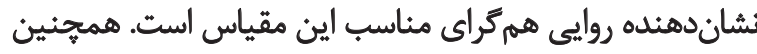

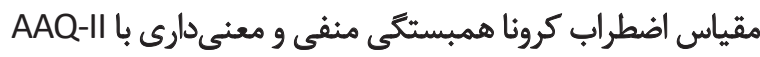

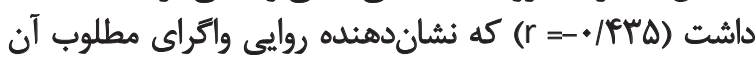




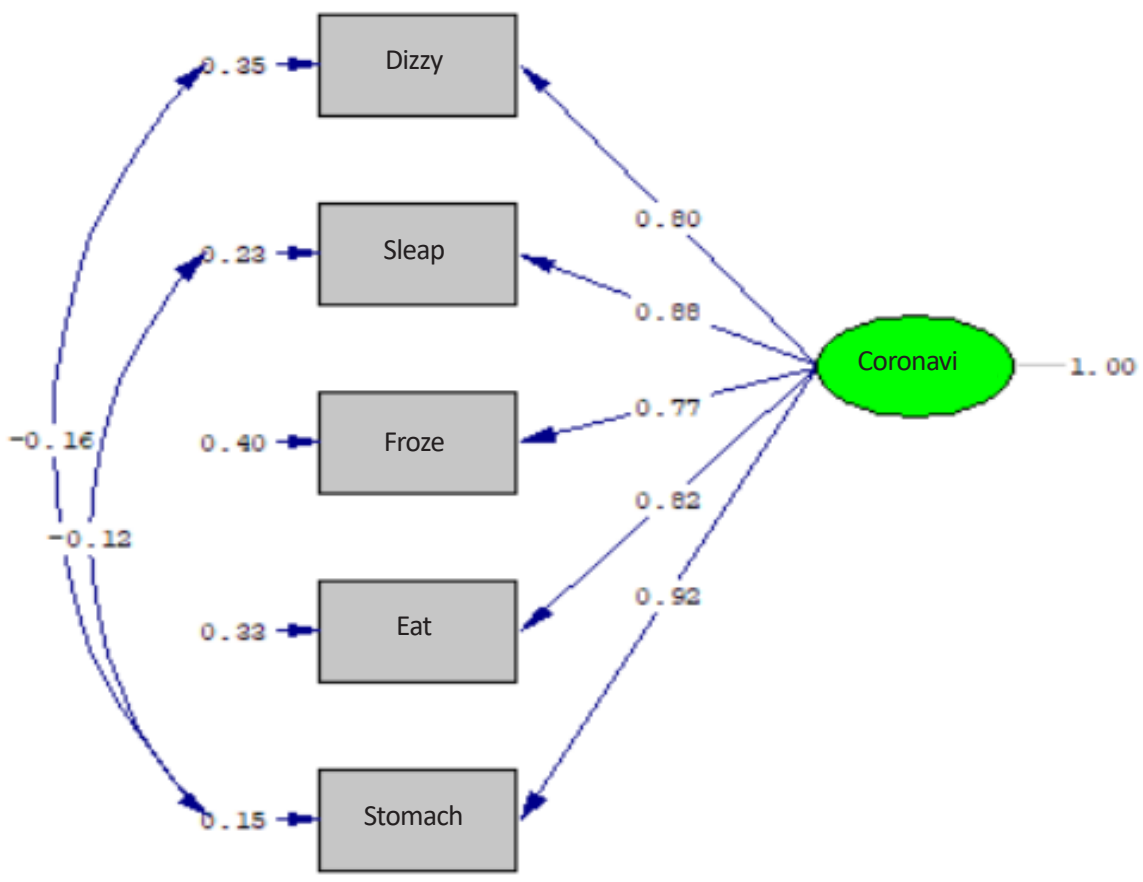

Chi-Square=4. 83, df=3, P-value=0. 18468, RMEA=0.043

به منظور بررسي روايي سازه از تحليل عاملي تأييدى استفاده

با توجه به همه كيرى ويروس كرونا در سرثاسر جهان و اثرات

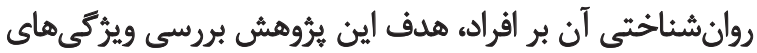

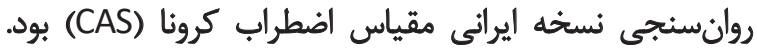

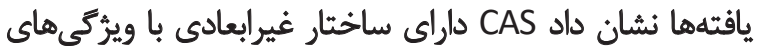

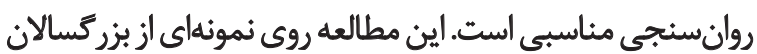

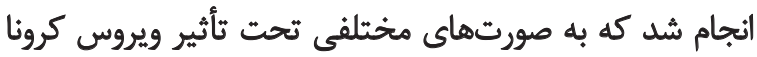

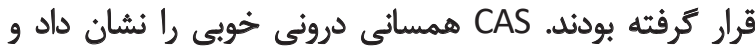

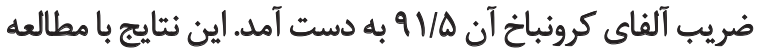

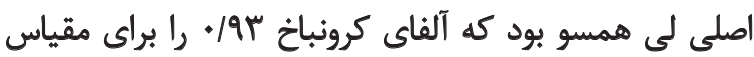

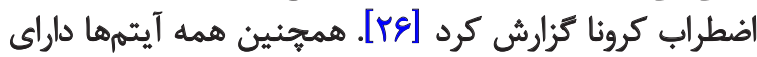

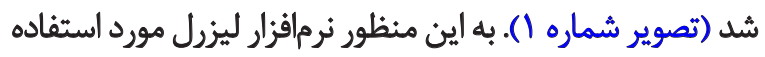

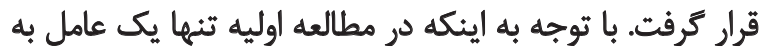

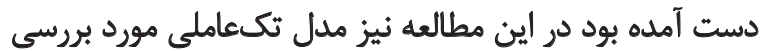

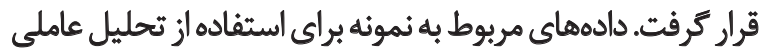

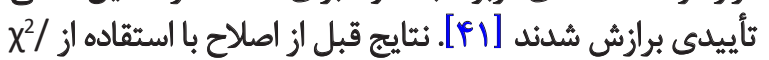

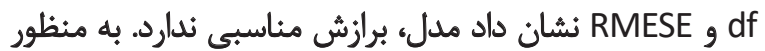

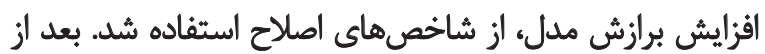

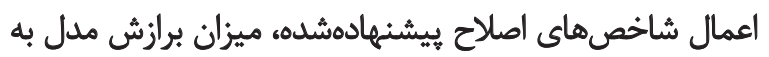

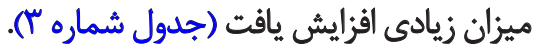




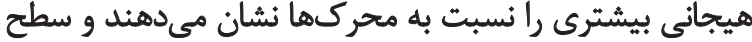

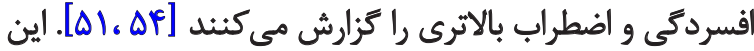

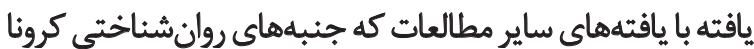

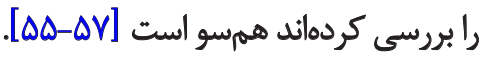

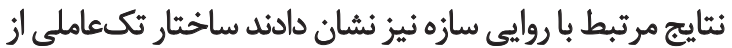

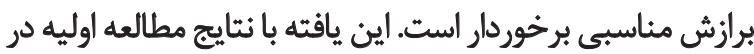

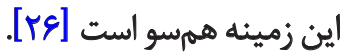

\section{نتيجلمَيرى}

مطالعه فعلى مقياس ينجسؤالى اضطراب كرونا را در نمونه

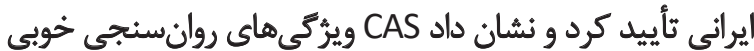

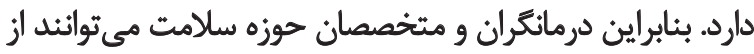

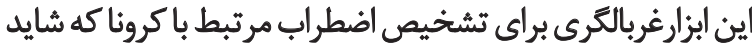
مامها ادامه داشتئه باشد، استفاده كنيند.

اين مطالعه داراى محدوديتهايى بود؛ اول اينكه نمونه ئيرى به

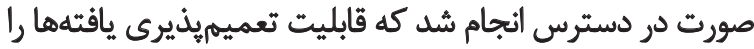

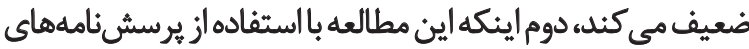

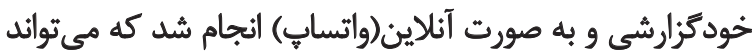

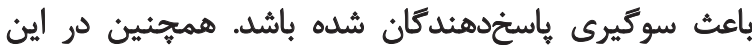

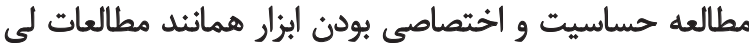

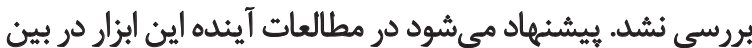

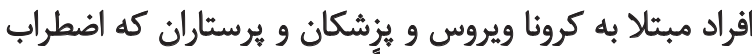

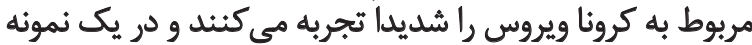

بزرى با تعيين حساسيت و اختصاصى بودن آن بررسى شود.

\section{مالاحظات اخلاقى}

\section{ييروى از اصول اخلاق يثروهش}

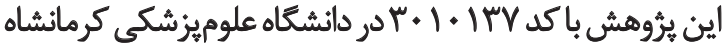

تصويب شده است.

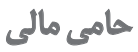

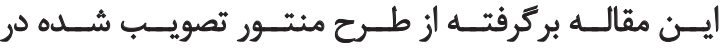

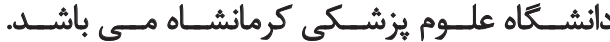

$$
\text { مشاركت نويسندكًان }
$$

مفهـوم سـازى: دكتـر على اكبرفروغـى، محسـن محمديور؛

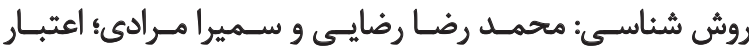

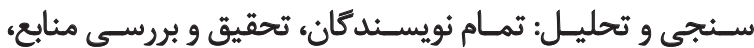

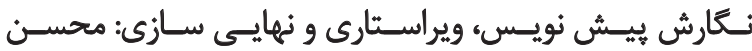

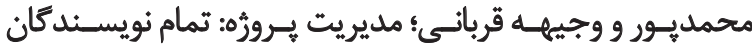

بروايي محتواي حُوبيى بودند كه نشانكُ اين است كه تمام سؤالات

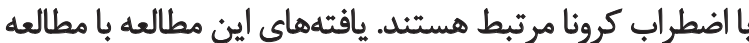

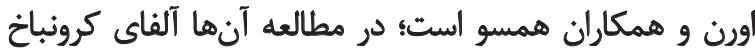

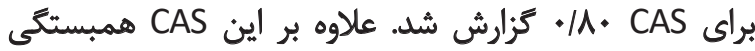

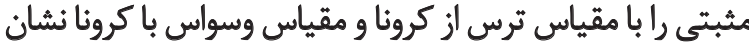

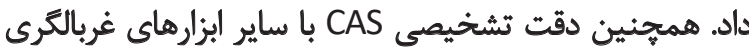

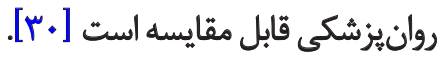

در اين مطالعه به منظور بررسى روايى همركرا از مقياس ترس

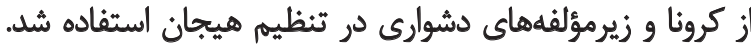

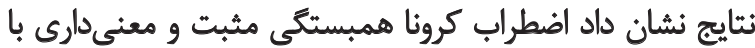

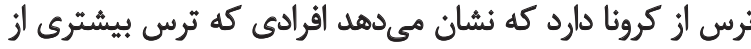

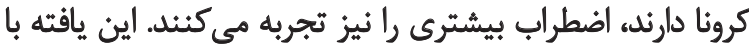

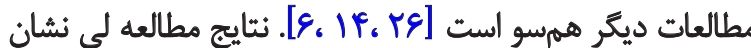

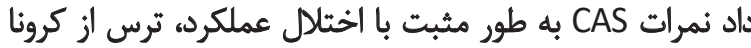

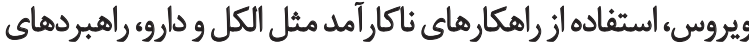

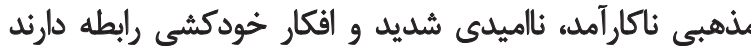

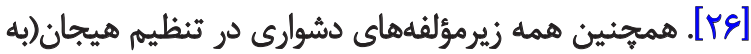

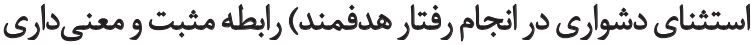

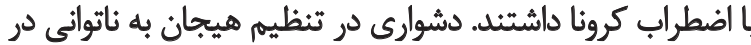

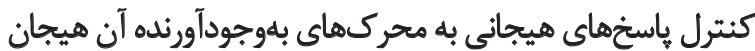

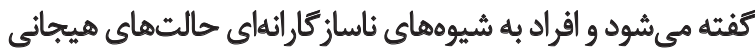

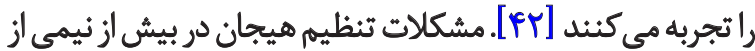

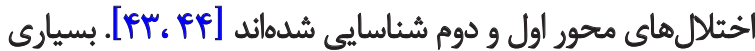

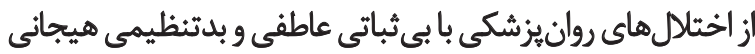

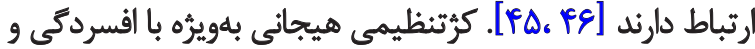

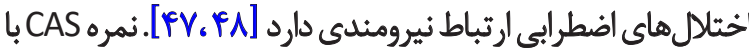

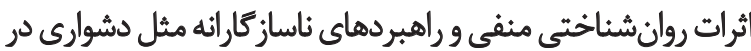

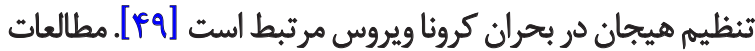

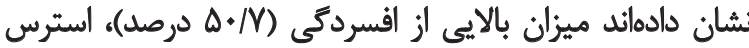

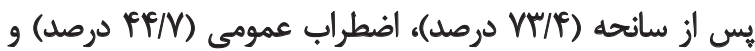

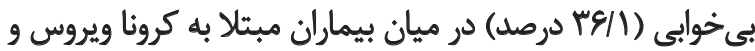

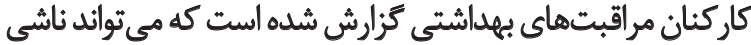

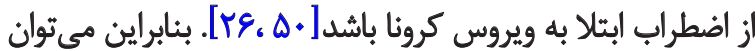

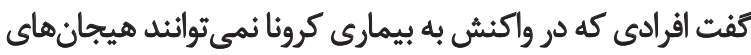
خود را تعديل كنثلد، اضطراب بيشترى را تجربه خواهند كردي به منظور بررسى روايى واكرا نيز از نسخه • اسؤالى برسش تئامه

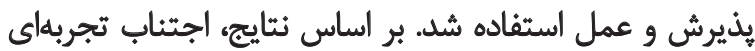

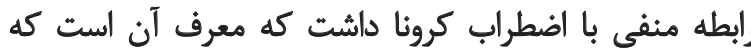

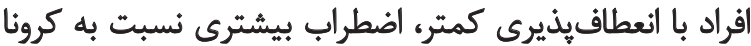

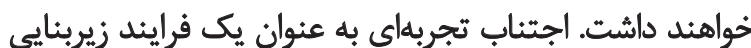

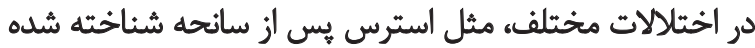

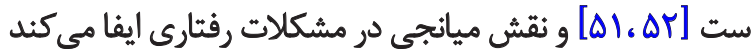

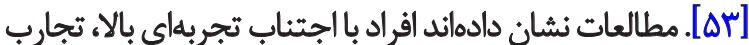




$$
\text { تعارض مثاقع }
$$

بنابراظهار نويسئدكان اين مقاله تعارض منافع ندارد.

$$
\text { تشكر و قدردانى }
$$

بدينوسيله از معاونت محترم تحقيقات و فناورى دانشكاه

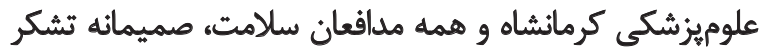

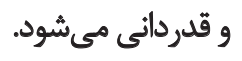




\section{References}

[1] Sohrabi C, Alsafi Z, O'Neill N, Khan M, Kerwan A, Al-Jabir A et al. World Health Organization declares global emergency: A review of the 2019 novel Coronavirus (COVID-19). International Journal of Surgery. 2020; 76:71-6. [DOI:10.1016/j.ijsu.2020.02.034] [PMID] [PMCID]

[2] Louie PK, Harada GK, McCarthy MH, Germscheid N, Cheung JPY, Neva MH, et al. The impact of COVID-19 pandemic on spine surgeons worldwide. Global Spine Journal. 2020; 10(5):534-52. [DOI:10.1177/2192568220925783] [PMID] [PMCID]

[3] World Health Organization. Coronavirus Disease (COVID-19) pandemic. Geneva: World Health Organization; 2020. https:// www.who.int/emergencies/diseases/novel-coronavirus2019?gclid=EAIaIQobChMI5uq76MDD7gIVeYFQBh2HRA5QE AAYASAAEgJXR_D_BwE

[4] Xiang YT, Yang Y, Li W, Zhang L, Zhang Q, Cheung T, et al. Timely mental health care for the 2019 novel coronavirus outbreak is urgently needed. The Lancet Psychiatry. 2020; 7(3):228-9. [DOI:10.1016/S2215-0366(20)30046-8]

[5] Asgari Z, Naghavi A. [Explaining post-traumatic growth: Thematic synthesis of qualitative research (Persian)]. Iranian Journal of Psychiatry and Clinical Psychology. 2019; 25(2):222-34. [DOI:10.32598/ijpcp.25.2.222]

[6] Ahorsu DK, Lin C-Y, Imani V, Saffari M, Griffiths MD, Pakpour $\mathrm{AH}$. The Fear of COVID-19Scale: Development and initial validation. International Journal of Mental Health and Addiction. 2020. [DOI:10.1007/s11469-020-00270-8]

[7] Dong L, Hu S, Gao J. Discovering drugs to treat Coronavirus Disease 2019 (COVID-19). Drug Discoveries \& Therapeutics. 2020; 14(1):58-60. [DOI:10.5582/ddt.2020.01012] [PMID]

[8] Wang D, Hu B, Hu C, Zhu F, Liu X, Zhang J, et al. Clinical characteristics of 138 hospitalized patients with 2019 novel coronavirus-infected pneumonia in Wuhan, China. JAMA. 2020; 323(11):1061-9. [DOI:10.1001/jama.2020.1585] [PMID] [PMCID]

[9] Chong MY, Wang WC, Hsieh WC, Lee CY, Chiu NM, Yeh WC, et al. Psychological impact of severe acute respiratory syndrome on health workers in a tertiary hospital. The British Journal of Psychiatry. 2004; 185(2):127-33. [DOI:10.1192/bjp.185.2.127] [PMID]

[10] Wheaton MG, Abramowitz JS, Berman NC, Fabricant LE, Olatunji BO. Psychological predictors of anxiety in response to the H1N1(swine flu) pandemic. Cognitive Therapy and Research. 2012; 36(3):210-8. [DOI:10.1007/s10608-011-9353-3]

[11] Wu P, Fang Y, Guan Z, Fan B, Kong J, Yao Z, et al. The psychological impact of the SARS epidemic on hospital employees in China: Exposure, risk perception, and altruistic acceptance of risk. The Canadian Journal of Psychiatry. 2009; 54(5):302-11. [DOI:10.1 177/070674370905400504] [PMID] [PMCID]

[12] Yip PSF, Cheung YT, Chau PH, Law YW. The impact of epidemic outbreak: The case of Severe Acute Respiratory Syndrome (SARS) and suicide among older adults in Hong Kong. Crisis. 2010; 31(2):86-92. [DOI:10.1027/0227-5910/a000015] [PMID]

[13] Dong XY, Wang L, Tao YX, Suo XL, Li YC, Liu F, et al. Psychometric properties of the anxiety inventory for respiratory disease in patients with COPD in China. International Journal of Chronic Obstructive Pulmonary Disease. 2017; 12:49-58. [DOI: 10.2147/ COPD.S117626
[14] Harper CA, Satchell LP, Fido D, Latzman RD. Functional fear predicts public health compliance in the COVID-19 pandemic International Journal of Mental Health and Addiction. 2020 [DOI:10.1007/s11469-020-00281-5] [PMID] [PMCID]

[15] Banerjee D. The COVID-19 outbreak: Crucial role the psychiatrists can play. Asian Journal of Psychiatry. 2020; 50:102014. [DOI:10.1016/j.ajp.2020.102014] [PMID] [PMCID]

[16] Moghanibashi-Mansourieh A. Assessing the anxiety leve of Iranian general population during COVID-19 outbreak. Asian Journal of Psychiatry. 2020; 51:102076. [DOI:10.1016/j. ajp.2020.102076] [PMID] [PMCID]

[17] Bajema KL, Oster AM, McGovern OL, Lindstrom S, Stenger MR, Anderson TC, et al. Persons evaluated for 2019 novel coronavirus - United States, January 2020. MMWR. Morbidity and Mortality Weekly Report. 2020; 69(6):166-70. [DOI:10.15585/mmwr mm6906e1] [PMID] [PMCID]

[18] Chan JFW, Yip CC-Y, To KKW, Tang THC, Wong SCY, Leung K-H, et al. Improved molecular diagnosis of COVID-19 by the novel, highly sensitive and specific COVID-19-RdRp/Hel real-time reverse transcription-PCR assay validated in vitro and with clinical specimens. Journal of Clinical Microbiology. 2020; 58(5):e00310-20. [DOI:10.1128/JCM.00310-20] [PMID] [PMCID]

[19] Pérez-Fuentes MDC, Molero Jurado MDM, Oropesa Ruiz NF, Martos Martínez Á, Simón Márquez MDM, Herrera-Peco I, et al. Questionnaire on Perception of Threat from COVID-19. Journal of Clinical Medicine. 2020; 9(4):1196. [DOI:10.3390/jcm9041196] [PMID] [PMCID]

[20] Ayora AF, Soler LM, Gasch AC. Análise de dois questionários sobre a qualidade de vida em pacientes com doença pulmonar obstrutiva crônica. Revista Latino-Americana de Enfermagem. 2019; 27:e3148. [DOI:10.1590/1518-8345.2624.3148] [PMID] [PMCID]

[21] Yohannes AM, Willgoss TG, Fatoye FA, Goldbart J. Validity and reliability of the anxiety inventory for respiratory disease scale in patients with copd, in c42. Creating The Evidence: Generating Tools And Developing Methodologies For Pulmonary And Critical Care Research. American Thoracic Society; 2013.

[22] Taylor S. The psychology of pandemics: Preparing for the next global outbreak of infectious disease. United Kingdom: Cam bridge Scholars Publishing; 2019. https:/ / www.cambridgescholars.com/product/978-1-5275-3959-4

[23] Kumar A, Somani A. Dealing with Corona virus anxiety and OCD. Asian Journal of Psychiatry. 2020; 51:102053. [DOI:10.1016/j.ajp.2020.102053] [PMID] [PMCID]

[24] Lee SA. How much "Thinking" about COVID-19 is clinically dysfunctional? Brain, Behavior, and Immunity, 2020; 87:97-8. [DOI:10.1016/j.bbi.2020.04.067] [PMID] [PMCID]

[25] Hamza Shuja K, Aqeel M, Jaffar A, Ahmed A. COVID-19 pandemic and impending global mental health implications. Psychiatria Danubina. 2020; 32(1):32-5. [DOI:10.24869/psyd.2020.32] [PMID]

[26] Lee SA. Coronavirus Anxiety Scale: A brief mental health screener for COVID-19 related anxiety. Death Studies. 2020; 44(7):393-401. [DOI:10.1080/07481187.2020.1748481] [PMID]

[27] Asmundson GJ, Taylor S. Coronaphobia: Fear and the 2019nCoV outbreak. Journal of Anxiety Disorders. 2020; 70:102196 [DOI:10.1016/j.janxdis.2020.102196] [PMID] [PMCID] 
[28] Lee SA. Replication analysis of the Coronavirus Anxiety Scale Dusunen Adam: The Journal of Psychiatry and Neurological Sciences. 2020; 33. [DOI:10.14744/DAJPNS.2020.00079]

[29] Lee SA, Mathis AA, Jobe MC, Pappalardo EA. Clinically significant fear and anxiety of COVID-19: A psychometric examination of the Coronavirus Anxiety Scale. Psychiatry Research. 2020; 290:113112. [DOI:10.1016/j.psychres.2020.113112] [PMID] [PMCID]

[30] Evren C, Evren B, Dalbudak E, Topcu M, Kutlu N. Measuring anxiety related to COVID-19: A Turkish validation study of the Coronavirus Anxiety Scale. Death Studies. 2020:1-7. [DOI:10.1080 /07481187.2020.1774969] [PMID]

[31] Shah R, Goldstein SM. Use of structural equation modeling in operations management research: Looking back and forward. Journal of Operations management. 2006; 24(2):148-69. [DOI:10.1016/j.jom.2005.05.001]

[32] Kline RB. Principles and practice of structural equation modeling. $4^{\text {th }}$ ed. New York: Guilford publications; 2015. https:// www.guilford.com/books/Principles-and-Practice-of-Structural-Equation-Modeling/Rex-Kline/9781462523344

[33] Guillemin F, Bombardier C, Beaton D. Cross-cultural adaptation of health-related quality of life measures: literature review and proposed guidelines. Journal of Clinical Epidemiology. 1993; 46(12):1417-32. [DOI:10.1016/0895-4356(93)90142-N]

[34] Gjersing L, Caplehorn JRM, Clausen T. Cross-cultural adaptation of research instruments: language, setting, time and statistical considerations. BMC Medical Research Methodology. 2010; 10:13. [DOI:10.1186/1471-2288-10-13] [PMID] [PMCID]

[35] Gratz KL, Roemer L. Multidimensional assessment of emotion regulation and dysregulation: Development, factor structure, and initial validation of the difficulties in emotion regulation scale. Journal of psychopathology and behavioral assessment. 2004; 26(1):41-54. [DOI:10.1023/B:JOBA.0000007455.08539.94]

[36] Khanzadeh M, Saeediyan M, Hosseinchari M, Edrissi F. [Factor structure and psychometric properties of difficulties in emotional regulation scale(Persian)]. International Journal of Behavioral Sciences. 2012; 6(1):87-96. http://www.behavsci.ir/article_67768_01 a5f82ae30d4cbf3dd322fd6618c7eb.pdf

[37] Bond FW, Hayes SC, Baer RA, Carpenter KM, Guenole N, Orcutt HK, et al. Preliminary psychometric properties of the Acceptance and Action Questionnaire-II: A revised measure of psychological inflexibility and experiential avoidance. Behavior Therapy. 2011; 42(4):676-88. [DOI:10.1016/j.beth.2011.03.007] [PMID]

[38] Kleszcz B, Dudek JE, Białaszek W, Ostaszewski P, Bond F. The psychometric properties of the Polish version of the Acceptance and Action Questionnaire-II(AAQII). Studia Psychologiczne. 2018; 56(1):1-19. http://research.gold.ac.uk/id/eprint/24769/

[39] Abasi E, Fti L, Molodi R, Zarabi H. [Psychometric properties of Persian version of acceptance and action questionnaireII(Persian)]. Journal Mamagement System 2013; 3(2):65-80. http://jpmm.miau.ac.ir/article_61.html

[40] Tabachnick BG, Fidell LS. Using multivariate statististics. Boston: Allyn and Bacon; 2001. https://books.google.com/books/ about/Using_Multivariate_Statistics.html?id=IVtqAAAAMAAJ

[41] Schermelleh-Engel K, Moosbrugger H, Müller H. Evaluating the fit of structural equation models: Tests of significance and descriptive goodness-of-fit measures. Methods of Psychological
Research Online. 2003; 8(2):23-74. https://psycnet.apa.org/record/2003-08119-003

[42] Gross JJ, Jazaieri H. Emotion, emotion regulation, and psychopathology: An affective science perspective. Clinical Psychological Science. 2014; 2(4):387-401. [DOI:10.1177/2167702614536164]

[43] Gross JJ, Muñoz RF. Emotion regulation and mental health. Clinical Psychology: Science and practice. 1995; 2(2):151-64. [DOI:10.1111/j.1468-2850.1995.tb00036.x]

[44] Repetti RL, Taylor SE, Seeman TE. Risky families: Family social environments and the mental and physical health of offspring. Psychological Bulletin. 2002; 128(2):330. [DOI:10.1037/00332909.128.2.330] [PMID]

[45] Ochsner KN, Gross JJ. The neural architecture of emotion regulation. Handbook of Emotion Regulation. 2007; 1(1):87-109. [DOI:10.1111/j.1751-9004.2007.00005.x]

[46] Silk JS, Steinberg L, Morris AS. Adolescents' emotion regulation in daily life: Links to depressive symptoms and problem behavior. Child Development. 2003; 74(6):1869-80. [DOI:10.1046/ j.1467-8624.2003.00643.x] [PMID]

[47] Phillips ML, Drevets WC, Rauch SL, Lane R. Neurobiology of emotion perception I: The neural basis of normal emotion perception. Biological psychiatry. 2003; 54(5):515-28. [DOI:10.1016/ S0006-3223(03)00171-9]

[48] Mennin DS. Emotion regulation therapy for generalized anxiety disorder. Clinical Psychology \& Psychotherapy: An International Journal of Theory \& Practice. 2004; 11(1):17-29. [DOI:10.1002/ cpp.389]

[49] Mohammadpour M, Ghorbani V, Khoramnia S, Ahmadi SM, Ghvami M, Maleki M. anxiety, self-compassion, gender differences and COVID-19: Predicting self-care behaviors and fear of COVID-19 based on anxiety and self-compassion with an emphasis on gender differences. Iranian Journal of Psychiatry. 2020; 15(3):213. [DOI:10.18502/ijps.v15i3.3813] [PMID] [PMCID]

[50] Lai J, Ma S, Wang Y, Cai Z, Hu J, Wei N, et al. Factors associated with mental health outcomes among health care workers exposed to coronavirus disease 2019. JAMA Network Open. 2020; 3(3):e203976. [DOI:10.1001/jamanetworkopen.2020.3976] [PMID] [PMCID]

[51] Tull MT, Gratz KL, Salters K, Roemer L. The role of experiential avoidance in posttraumatic stress symptoms and symptoms of depression, anxiety, and somatization. The Journal of Nervous and Mental Disease. 2004; 192(11):754-761. [DOI:10.1097/01. nmd.0000144694.30121.89] [PMID]

[52] Kashdan TB, Barrios V, Forsyth JP, Steger MF. Experiential avoidance as a generalized psychological vulnerability: Comparisons with coping and emotion regulation strategies. Behaviour Research and Therapy. 2006; 44(9):1301-20. [DOI:10.1016/j. brat.2005.10.003] [PMID]

[53] Kingston J, Clarke S, Remington B. Experiential avoidance and problem behavior: A mediational analysis. Behavior Modification. 2010; 34(2):145-63. [DOI:10.1177/0145445510362575] [PMID]

[54] Sloan DM. Emotion regulation in action: Emotional reactivity in experiential avoidance. Behaviour Research and Therapy. 2004; 42(11):1257-70. [DOI:10.1016/j.brat.2003.08.006] [PMID]

[55] Liu N, Zhang F, Wei C, Jia Y, Shang Z, Sun L, et al. Prevalence and predictors of PTSS during COVID-19 outbreak in China hardest-hit areas: Gender differences matter. Psychiatry Research. 
2020; 287:112921. [DOI:10.1016/j.psychres.2020.112921] [PMID] [PMCID]

[56] Li S, Wang Y, Xue J, Zhao N, Zhu T. The impact of COVID-19 epidemic declaration on psychological consequences: A study on active Weibo users. International Journal of Environmental Research and Public Health. 2020; 17(6):2032. [DOI:10.3390/ijerғ ph17062032] [PMID] [PMCID]

[57] Zhang C, Yang L, Liu S, Ma S, Wang Y, Cai Z, et al. Survey of insomnia and related social psychological factors among medical staffs involved with the 2019 novel coronavirus disease outbreak. Frontiers in Psychiatry. 2020; 11:306. [DOI:10.3389/fpp syt.2020.00306] [PMID] [PMCID] 
This Page Intentionally Left Blank 